26th Forum on Information and Knowledge Proceedings

\title{
Big Data in the Humanities: New Interdisciplinary Opportunities and New Challenges for Data Management
}

\author{
R. Michael Feener ${ }^{1 *}$, Noboru Ishikawa ${ }^{2} \&$ Patrick Daly $^{3}$ \\ 1 Center for Southeast Asian Studies, Kyoto University \\ E-mail: feener@,cseas.kyoto-u.ac.jp \\ 2 Center for Southeast Asian Studies, Kyoto University \\ E-mail: ishikawa@cseas.kyoto-u.ac.jp \\ 3 Earth Observatory of Singapore, Nanyang Technological University \\ E-mail: patrickdaly@ntu.edu.sg
}

Recent developments have made technologies such as LiDAR and photogrammetry visualizations more widely accessible to scholars in the humanities. This, in turn, is currently facilitating the production of larger and more complex data sets than those that have heretofore been associated with Digital Humanities. This new turn has stimulated innovative work in a number of fields, but also given rise to new challenges for researchers, IT departments, and university libraries. This paper highlights a number of such issues related to the management of research data with reference to the work the Maritime Asia Heritage Survey (MAHS) based at Kyoto University's Center for Southeast Asian Studies. The MAHS uses traditional archaeological survey techniques while also incorporating data capture through newer technologies of LiDAR scanning, RTK/GIS, digital photography and video, photogrammetry, $\mathrm{CAD}$, and 3D modelling to produce interactive visualizations, IIIF deep-zoom digitized manuscripts, oral history recordings, architectural plans and elevations, and orthophotomaps integrated into robust records in an Arches database. Establishing this project at Kyoto University has highlighted the importance of developing new hardware infrastructure, policy guidelines, and support for the long-term management of the digital knowledge resources as crucial aspects of the institutional infrastructure for research universities in the future.

Keywords: digital humanities, big data, archaeology, history, heritage, open access, Maritime Asia Heritage Survey

\section{Introduction}

Discussions of 'Digital Humanities' had - until recently - been somewhat separate from those on 'big data'. With developments in new technologies including more widely accessible means for LiDAR and photogrammetry visualizations, more work in the humanities Is producing and working with larger and more 
complex data sets than ever before. This has not only facilitated stimulating new work in a number of fields, but also given rise to new challenges for researchers, IT departments, and university libraries. Today, I would like to highlight a number of such issues related to the management of research data with reference to the work of my colleagues and I on the Maritime Asia Heritage Survey (MAHS) [1][2], based at Kyoto University's Center for Southeast Asian Studies.

\section{Maritime Asia Heritage Survey}

The Maritime Asia Heritage Survey works to discover, inventory, and digitally document critically endangered heritage sites in coastal regions across Southern Asia and to create an open-access and permanently preserved digital archive of archaeological, historical, anthropological, architectural, and literary source material documentation of the heritage of this dynamic region of cultural and commercial circulation with fieldwork in the Maldives, Indonesia, Sri Lanka, and Vietnam [3]. The MAHS is committed to both producing new forms of digital documentation and facilitating the open accessibility and secure long-term preservation of that material. These goals are pursued through project design, digital archive construction, documentation standardization to produce robust records of sites, structures, and objects across the region as well as the creation of new digital assets based upon this primary data that will be integrated into the online archive we are building.
As the countries where we work have limited capacity and access to technologies needed to produce high quality digital heritage assets, our project provides resources to local field teams and invests significantly in capacity building thus helping to establish solid foundations upon which future work can be conducted that would make possible the open-ended expansion of the digital archive. Our field teams are not only trained in the technical operation of equipment, but also in the project's standardized methodology of using it to collect data on sites, structures, and objects. Each in-country field team also has a full-time data wrangler responsible for organizing all the files created in the course of the survey work, and organizing the upload of data to the cloud. After that, the Kyoto-based team at the MAHS Digital Heritage Documentation Lab works with the data to produce 3D models, maps, and other forms of visualization as well as the detailed records that integrate multi-media resourced into the project database.

The MAHS field teams implement a standardized set of procedures that uses traditional archaeological survey techniques as well as incorporating newer technologies of LiDAR scanning, RTK/GIS, digital photography and video, photogrammetry, $\mathrm{CAD}$, and 3D modelling to produce interactive visualizations, IIIF deep-zoom digitized manuscripts, oral history recordings, architectural plans and elevations, and orthophotomaps integrated into robust records in an Arches database. Our project thus pursues a model of work in the digital 
humanities that move considerably beyond established 'first generation' projects of digitizing existing analog materials. For, in addition to things like digitizing local manuscript materials, our project also generates a massive amount of complex data in the course of creating new digital heritage assets in the form of LiDAR point clouds, 3D models and other resources that were born digital. In the process we generate files in a number of different formats, including .csv, .xlsx, .sql, .pdf, .jpg, tiff, .png, mp4, .e57, .las, .lps, .lsproj, .aprx, .shp, .dwg, . dxf, .psx, .rcproj, .3ds, obj, .ply, and .kmz. Over the past year we have created 22,100 new records and their associated digital heritage assets to generate a large digital archive of nearly $2 \mathrm{~TB}$.

\section{Open-Access Dataset}

Our online digital archive is designed to be integrated with multimedia digital assets and connected meta-data in ways that facilitate dynamic search results and focused cross-referencing in ways that can support practical usage as we well as the formulation and exploration of new research questions. Arches is an open-source software package that was developed by the Getty Conservation Institute and the World Monuments Fund [4]. This GIS referenced database architecture uses a customizable framework of 'resource graphs.' Our project has developed this so as to be able to include not only the kinds of sites and structures for which the database was originally designed, but also for other kinds of record types which we have designed to accommodate and integrate material ranging from individual pottery sherds to complex 3D structural models produced through a combination of laser scanning and photogrammetry.

Arches is a potentially powerful inventory and data management tool, made even more attractive for projects such as this in being an open-source platform. Here, however, we need to recognize the complexities that come with anything designated as 'free' in the digital ecosystem that we now inhabit. In his Pre-History of the Cloud, Tung-Hui Hu called attention to the frequent and disconcerting slippage between libertarian hacker ideologies of 'free speech' and neo-liberal incentives of 'free beer' in the very DNA of the internet [5]. There are, however, yet other senses in which 'free' can be understood and experienced in cyberspace. Prospective Arches users need to bear in mind that it is 'free' only in the sense that say a puppy from a neighbor's litter is free - and thus it requires appropriate funding provisions in place to contract specialized developer support this platform which we accounted for in our grant funding proposal.

The database serves as the anchor for records that include a combination of field documentation and new digital heritage assets created by the MAHS Lab Team: 3D models, orthophotomaps, CAD site plans and architectural drawings, deep-zoom digitized manuscripts, oral history interviews and other video materials, as well as reference materials to facilitate contextualization, including an 
illustrated glossary, a virtual library of open-access publications, an interactive 3D timeline, and a blog. In addition to our project website, we have set up MAHS channels on YouTube and Sketchfab [7], as well as new social media profiles to publicize the work of the project on Instagram, Twitter, LinkedIn and Facebook.

Our online archive is open-access and we are fortunate to have found in the Arcadia Fund financial supporters for our work who are themselves fully committed to open-access as a foundational principle [6]. We are thus able to ensure that all digital heritage assets created in the course of this project will be made open-access available through our website based on the servers of Kyoto University. Anyone can access the records in the Arches database, and the contextualizing reference material that we have specially produced in the course of the project there, including an annotated timeline, an illustrated guideline of local-language ornamental and architectural terminology, and a virtual library. Digital heritage assets including 3D models and video are publicly available on SketchFab and YouTube, while full registered point clouds for our LiDAR data is available for free download at OpenHeritage3D via Creative Commons Licensing (Attribution-NoDerivatives 4.0 International) [8].

Of course, scholars have long mused about how their work might be used by posterity, but here we find ourselves thrust into new kinds of consideration of our scholarly obligations to both the past that we endeavor to preserve, and the future accessibility of what we produce.

\section{Long-term Digital Archiving}

Consideration of the implications of this raise questions about the nature of archive building in the twenty-first century that go beyond earlier discussions of digitizing sources. It is not simply a matter of the 'migration' of a traditional repository of cultural artefacts to a digital platform (10), but rather the shaping of new kinds of archives - allowing for new forms of engagement which both consider multiple kinds of usages by diverse users in the near present and recognize that there may be a new range of unexpected usage in the future.

Earlier conversations on Digital Humanities focused on issues arising from the work of 'scanning the archive' in ways that tended to cast the situation as one of a stable body of material that was worked into new media, which brought with it various possibilities and/or problems - depending on the commentator. Then, as 'Big Data' sets came into being, a new host of issues involved with 'archiving the digital' came to the fore. Our project, while sharing features that place it within both these lines of conversation also enters into another area which deserves critical attention: the shaping of a multimedia digital archive that incorporates existing digital records, scanned paper documents, primary field survey data, and a growing collection of new digital assets generated out of this.

In addition to ensuring open access to all of our data, the MAHS also provides for the 
long-term stable preservation of its digital archive. Full packages all digital heritage assets created by the project and accompanying metadata are compiled and transferred to both permanent data repositories on an annual basis. To ensure long-term future accessibility of all digital assets, metadata is consistently formatted, and each batch deposit includes a accumulative 'read-me' file containing a detailed asset inventory, general rights for reuse, contact information, a recommended citation, and a synopsis of the project. Files are saved in both their current application formats and also exported into the most widely used and durable file types available for various kinds of data - such as .pdfs generated for each database record. Building upon the pilot phase of this project based at the Oxford Centre for Islamic Studies, we have been able to deposit one copy of our project data in the Oxford Research Archive, which assigns a permanent URL for data deposits for our project and provides long-term curation in the Bodleian Libraries archival store. Since moving the project here to Kyoto University, we have been struggling to establish a similar arrangement to allow for the data we generate to be responsibly preserved at our primary institutional home [9].

The work of constructing a complex, multimedia archive of diverse digital assets entails complex institutional, as well as intellectual considerations. Beyond the first order issues of obtaining project funding, specialist recruitment, assembling IT infrastructure and managing logistics, a project such as this can pose challenges for which some traditional university structures are unprepared at present. A project such as ours thus both draws on the support of - and potentially drive changes to - other segments of the university system including the IT Office or Media Center and the University Library. There is thus a host of both intellectual and institutional issues to consider in relation to the emergence of a new digital information ecosystem.

Our project is amassing a large amount of data from diverse sources in the course of our project, and then working to make sure that it is both archived for long-term preservation and made freely accessible online to anyone who might be interested to use in ways that are most productive and meaningful to them. Though the primary source material is of kinds that might be traditionally associated with the fields of history and archaeology that are oriented toward understanding the past. However we also devote considerable critical thought toward issues related to the kinds of digital assets we create and how the material we generate through our work will be preserved, transmitted, used, and possibly transformed in new directions.

\section{Institutional Considerations}

As I hope to have shown in the brief introduction above to our work on the Maritime Asia Heritage survey, our massive data set incorporating a diverse and complex range of file types that provide for an innovative 'next generation' approach to the 
'digital humanities'. There are tremendous possibilities now for work such as this to position Kyoto University as a leader in the future of Area Studies Informatics, and to contribute to wider developments across other fields and disciplines. The overarching goal of our work is thus not only to document the endangered heritage of Maritime Southern Asia, but also more generally to assemble, preserve, and freely share a body of digital resources that can be dynamically engaged by anyone looking for resources to help them explore in new ways an open-ended range of questions.

In order to ensure that this work of the twenty-first century university is able to both make cutting-edge contributions today and provide a sustainable knowledge resource for use by future generations, researchers, funding agencies, universities, and governments will have to develop new frameworks of cooperation. In the case of the Maritime Asia Heritage Survey, the primary investigators have designed a project plan the provides its own specialist IT expertise and data management staff. We also took the initiative to obtain independent financial support for the project from an international funder who understands and is actively engaged in promoting the open-access digital environment of contemporary global scholarship. We are now looking forward to working more with Kyoto University to develop new hardware infrastructure, policy guidelines, and support for the long-term management of the new digital knowledge resources that will be a crucial aspect of the institutional infrastructure for research universities in the future.

\section{References}

[1] R. Michael Feener, Patrick Daly \& Nobo ru Ishikawa, "The Maritime Asia Heritage Su rvey,” IIAS Newsletter 88 (Spring 2021), htt ps://www.iias.asia/the-newsletter/article/maritim e-asia-heritage-survey

[2] MAHS Digital Heritage Documentation L $\mathrm{ab}$, https://maritimeasiaheritage.cseas.kyoto-u.ac. ip

[3] R. Michael Feener, Patrick Daly, Michael Frachetti, Ibrahim Mujah, Maida Irawani, Jo vial Pally Taran, Ahmad Zaki, Fathimath Ma asa, Mohamed Shamran, Multia Zahara, Mari yam Isha Azeez, Krisztina Baranyai, Paula L evick, Hala Bakheit, Jessica Rahardjo \& Gab riel Clark, "The Maldives Heritage Survey," Antiquity 95.381 (2021) DOI: https://doi.org/10. 15184/aqy.2021.45

[4] Arches Project, https://www.archesproject.org/

[5] Tung-Hui Hu, "A Prehistory of the Cloud, " Cambridge, MA: MIT Press, 2015, 121.

[6] Arcadia is a charitable fund of Lisbet Rausing and Peter Baldwin https://www.arcadiafund.org.uk/promoting-ope $\underline{\text { n-access }}$

[7] Sketchfab, https://sketchfab.com/blogs/com munity/documenting-and-digitally-preserving-th e-heritage-of-maritime-asia/

[8] OpenHeritage3D, https://openheritage3d.org /news.php? $\mathrm{p}=$ digitally-documenting-the-endange red-cultural-history-of-the-maldives

[9] Oxford Research Archive, https://www.bo dleian.ox.ac.uk/ora/about 